\title{
Superfilter Technique in SDN-Controlled Elastic Optical Networks [Invited]
}

\author{
F. Paolucci, F. Cugini, F. Fresi, G. Meloni, A. Giorgetti, N. Sambo, \\ L. Potí, A. Castro, L. Velasco, and P. Castoldi
}

\begin{abstract}
In flexigrid elastic optical networks, filtering cascade effects strongly affect the overall transmission performance. To avoid these detrimental impairments, each lightpath is typically configured, in an independent way, to encompass additional spectrum resources. Thus, the flat central region of the traversed filters are exploited and their transition region around the cutoff frequencies is avoided. However, handling lightpaths independently leads to less efficient spectrum utilization. In this study, we propose a novel technique, called superfilters, which enables different lightpaths, with different source-destination pairs, to coexist within the same flat region of a single filter configuration. That is, the technique consists of a pathcomputation strategy that applies differentiated configurations of lightpath traversed filters, which are decoupled from head-end lightpath configurations. Simulative transmission results are provided to assess the benefits of the proposed technique. Then, an experimental implementation is presented, including a software-defined networking implementation successfully applied to a flexigrid network testbed.
\end{abstract}

Index Terms-Bandwidth variable; BV-WSS; Cascade; Elastic; EON; Filter; Filtering; Flexigrid; Impairment; Routing and spectrum assignment; ROADM; RSA; Spectrum selective switches; WSS.

\section{INTRODUCTION}

Flexigrid elastic optical networks (EONs) have been recently introduced to provide efficient use of spectrum resources [1-3] . Spectrum efficiency is enabled by bandwidthvariable optical cross-connects (BV-OXCs, also called spectrum-selective switches), which are configured in order to minimize the spectrum reserved to each optical connection (i.e., lightpath). In particular, the frequency slot of a data connection (i.e., lightpath) is first computed and then configured in BV-OXCs as a variable amount of frequency slices, with a slot-width granularity of $12.5 \mathrm{GHz}[\underline{4}, \underline{5}]$. The path-computation process is responsible for defining the route and size of the frequency slot by considering both network-spectrum resources and the expected

Manuscript received July 7, 2014; revised October 15, 2014; accepted October 22, 2014; published December 8, 2014 (Doc. ID 216344).

F. Paolucci (e-mail: fr.paolucci@sssup.it), F. Fresi, A. Giorgetti, N. Sambo, and P. Castoldi are with Scuola Superiore Sant'Anna, Pisa, Italy.

F. Cugini, G. Meloni, and L. Potí are with CNIT, Pisa, Italy.

A. Castro and L. Velasco are with the Optical Communications Group (GCO), Universitat Politecnica de Catalunya (UPC), Barcelona, Spain

http://dx.doi.org/10.1364/JOCN.7.00A285 lightpath-transmission performance. Path computation can be performed by a path-computation element (PCE) or by a software-defined networking (SDN) controller. In both cases, path computation typically relies on three network databases. The first database consists of the trafficengineering database (TED), which includes the network topology and related available spectrum resources. The second one, called the label-switched path database (LSP-DB), is used when the stateful functionality is adopted. LSP-DB stores and maintains the route of the active lightpaths in the network. The third database includes transmission parameters and is used to estimate the lightpath quality of transmission (QoT) before the actual connection setup takes place. To perform lightpath QoT evaluation, the path computation has to account for transmitter and receiver characteristics as well as for impairments induced during signal propagation [6].

One of the most relevant degradations can be represented by filtering cascade effects [ㄱ, $\underline{3}$. Indeed, BV-OXCs include filters with nonideal shapes. In particular, the adopted filters present a central region with flat performance, but a nonideal transition region around the cutoff frequency [9]. Filter cascade effects may then strongly affect the overall lightpath optical reach or the maximum number of BV-OXCs that can be traversed with adequate QoT. To avoid detrimental filtering effects, a larger amount of spectrum could be reserved. Indeed, a large allocated spectrum enables the transmitted signal to avoid the transition region of the filters and to safely operate on a filter flat central region. However, this reduces the overall network spectrum efficiency.

In this study, we propose a novel technique, called superfilter, aimed at reducing these detrimental effects and improving overall network spectrum efficiency. A superfilter consists of the aggregation of multiple independent BV-OXC filter configurations related to different lightpaths that flow through common BV-OXC output ports. Superfilters are suitable for the SDN architecture thanks to the native capability of SDN to directly perform independent and differentiated filter configurations $[\underline{10}, \underline{11}]$.

Superfilters could also be implemented in the context of the generalized multiprotocol label switching (GMPLS)/ PCE architecture. However, this technique is not particularly suitable for GMPLS, since BV-OXC resources are reserved according to head-end lightpath parameters, making the aggregation (at the control-plane level) of 
frequency slots (i.e., filters) related to independent lightpaths awkward.

In this study, the superfilter technique is introduced, evaluated, and successfully implemented in a testbed controlled by a specifically enhanced SDN-based architecture.

\section{RELATED WORKS}

Several studies have addressed impairment-aware path computations and the analysis of the most relevant impairments to be considered for networking aspects. Surveys [12] and [13] and references therein represent valuable comprehensive sources of information. More recent studies also focused on high bit rates and coherent detection techniques. For example, in [14], modeling of $100-\mathrm{Gb} / \mathrm{s}$ transmission is reported including a Gaussian approximation to compute the bit-error rate (BER) of quadrature phaseshift keying (QPSK) signals. Other relevant recent studies include [15-19]. Specific focus on filtering cascade effects is provided in [20], where the propagation through multiple wavelength-selective switches (WSS) for a fixed grid (i.e., $50-\mathrm{GHz}$ spacing) is carefully evaluated through experiments and numerical simulations. In particular, [20] shows that passband effects enhanced by propagation through multiple nodes represent the most significant impairment induced by ROADMs and that filtering effects can become a major limiting factor for optical transmission. In [9], the actual shape of BV-OXC filters based on liquid crystal on silicon $(\mathrm{LCoS})$ is presented. The filter shape shows flat performance in the central region and nonideal transition region around the cutoff frequency. Transmission through these filters has been demonstrated in several studies. For example, [21] shows through experiments the relevant detrimental filtering penalty.

In impairment-aware path computations, detrimental filtering effects have to be carefully considered. Thus, a constraint on the maximum number of traversed filters has to be computed according to the signal bandwidth and the filter width, defined as a multiple of $12.5 \mathrm{GHz}$ (to be compliant with ITU-T recommendations [4]).

So far, such filter width has been strictly considered to be the frequency slot of each traversing channel. Instead, in this study we propose a superfilter technique that enables the decoupling between the filter-width configuration and the actual frequency slot of a lightpath.

The proposed technique is then implemented within an SDN-controlled optical network testbed. For additional details on basic extensions of the SDN architecture and OpenFlow (OF) protocol to account for specific optical parameters, including the flexible-grid utilization, we refer to the experimental studies on SDN solutions for optical networks presented in [22-25].

The superfilter technique is validated here on a network testbed employing a specific transmission technique, called time-frequency packing (TFP) $[10,26]$. TFP relies, besides soft detection techniques, on a skillful combination of pulse shaping, coding, the adoption of proper linear filtering, a proper trellis-based maximum a posteriori symbol detection strategy, and possibly on proper multiuser processing [26]. Such a combination enables significant transmission performance, e.g., in terms of spectral efficiency. However, it is important to highlight that the proposed superfilter technique can also be applied and validated with different transmission techniques and different filter bandwidth values (e.g., as in the case of [7]).

\section{SUPERFILTER TECHNIQUE}

A lightpath with bit rate $b_{r}$ and modulation format $m_{f}$ is considered. The lightpath is routed along path $p$. According to the considered node architecture (e.g., broadcast and select or switch and select [8]), either one or two filters per node are traversed. In either case, the number $N$ of filters traversed by the lightpath is identified. Filter bandwidth is configured according to the reserved spectrum resources, with a granularity imposed by the ITU-T flexigrid specifications, i.e., as a number $S$ of slices of width $12.5 \mathrm{GHz}$. Given the nonideal filter shapes, detrimental filtering cascade effects may be induced on the optical signal. Different values of $S$ can be typically considered for a lightpath according to the number $N$ of traversed filters. In particular, it is typically possible to identify a minimum value $S_{m}$ when up to $N_{f}$ filters are safely traversed (i.e., penalty may be introduced but margins are adequate and QoT is guaranteed). On the other hand, when $N>N_{f}$, to limit detrimental filtering effects and guarantee adequate QoT, values of $S$ larger than $S_{m}$ (i.e., $S_{M}>S_{m}$ ) are required.

Figure 1(a) shows an example of a flexigrid network. Three independent lightpaths, having different sourcedestination node pairs (i.e., B-V, A-W, C-Z), are considered. The three lightpaths are routed along the path D-Q. In this example, one filter per node is considered, and $N_{f}=4$ is assumed to guarantee adequate QoT when $S_{m}=2$ slices is considered. Thus, for $N$ larger than $N_{f}=4, S_{M}=3$ slices is required.

In the considered example of Fig. 1(a), signal A-W traverses a number $N$ of filters larger than $N_{f}$. Three different scenarios are then evaluated. For each scenario, the filter configuration traversed by each lightpath is shown in Figs. 1(b)-1(d).

In the first scenario, shown in Fig. 1(b), lightpaths B-V and C-Z safely traverse filters configured with $S_{M}=3$ and no detrimental filtering effects are introduced on these two signals. On the other hand, lightpath A-W traverses an excessive number of filters configured with $S=S_{m}\left(N>N_{f}\right)$. Thus, QoT is not guaranteed for lightpath $\mathrm{A}-\mathrm{W}$ and the scenario results are unfeasible.

In the second scenario, shown in Fig. 1(c), all lightpaths including A-W traverse filters are configured with $S=S_{M}$. This way, at the expense of additional allocated spectrum resources, adequate QoT is guaranteed to all lightpaths and all scenario results are feasible.

In the third scenario, shown in Fig. 1(d), the proposed superfilter technique is applied. By exploiting the common route of the three lightpaths, the sequence of nodes along E-P is configured with a unique filter bandwidth, which 

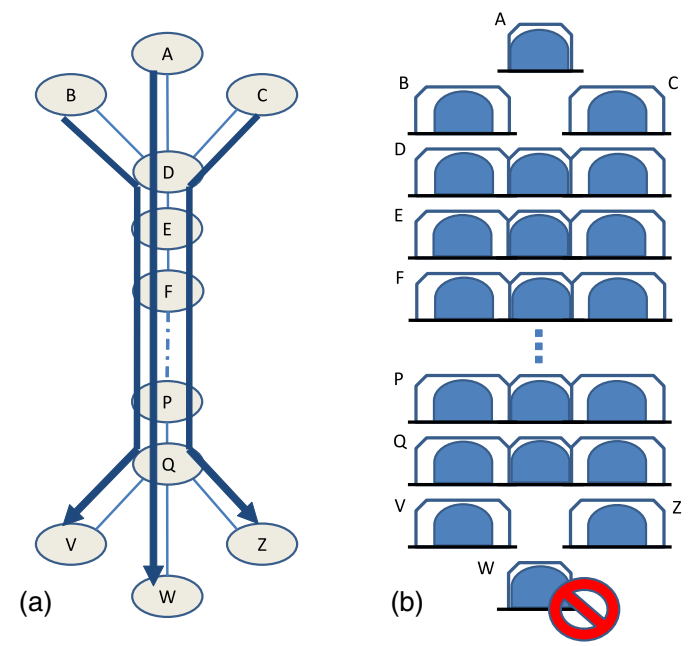

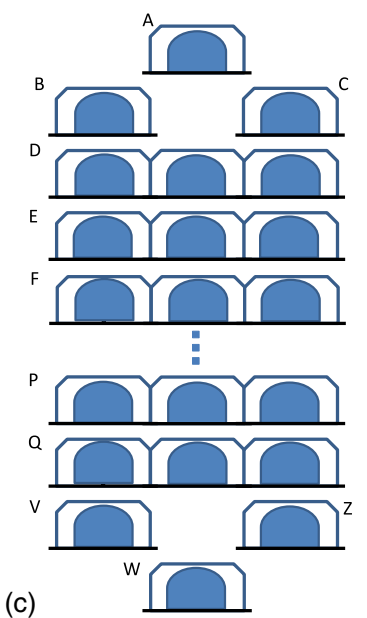

(c)

(d)

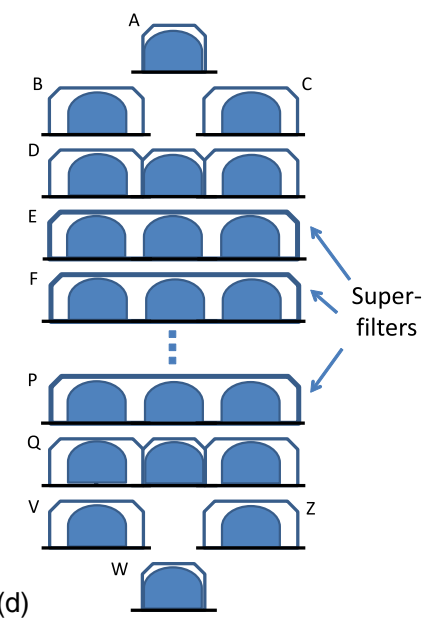

Fig. 1. (a) Reference network scenario including three lightpaths. (b)-(d) Configuration of all traversed filters. In particular, (b) lightpath A-W traverses filters configured with $S=S_{m}$ (e.g., $25 \mathrm{GHz}$ ). Lightpath A-W experiences excessive detrimental filtering effects. (c) Lightpath A-W traverses filters configured with $S=S_{M}$ (e.g., $37.5 \mathrm{GHz}$ ). QoT is guaranteed at the expense of additional reserved spectrum resources. (d) The superfilter technique is applied. A unique filter for all three ligtpaths is configured on E-P. These nodes do not induce detrimental filtering effects, and QoT on lightpath A-W is guaranteed even if it occupies only $S=S_{m}$ (e.g., $25 \mathrm{GHz}$ ).

encompasses all three signals (i.e., $S=S_{M}+S_{m}+S_{M}$, corresponding in this example to eight slices). With focus on lightpath A-W, end nodes A and W are configured with $S=S_{m}$. Node D (where a common outgoing link is selected among the three lightpaths) and node $\mathrm{Q}$ (where the last common link is used), given the considered broadcast and select node architecture, are also configured with $S=S_{m}$ for A-W resources. This way, lightpath A-W traverses four filters configured with $S=S_{m}$. However, the other filters along E-P are configured with $S=8$, a value significantly larger than $S_{m}=2$. This way, the constraint of $N_{f}$ is successfully addressed. Indeed, nodes along E-P do not induce detrimental filtering effects and the scenario exploiting superfilters has feasible results without requiring additional spectrum resources for lightpath A-W. Indeed, lightpath A-W occupies only two slices, saving one slice with respect to the previous feasible scenario of Fig. 1(c).

The proposed superfilter technique consists of a pathcomputation strategy that specifically accounts for the presence of other lightpaths and in particular for the actual configuration of BV-OXC filters in the network nodes. That is, the filter configuration is computed such that different lightpaths (e.g., with different source or destination nodes) can coexist within the same flat region of a single filter configuration. As a consequence, the technique decouples the filter configurations with respect to head-end lightpath configurations. The technique can be considered an extended application of the filter configuration typically applied to superchannels (i.e., a contiguous set of corouted optical signals having the same source-destination pair). In this study, common filter configurations are also applied to lightpaths having different source-destination pairs, exploiting their corouting along links and segments of the flexigrid optical network.

Differently with respect to traditional networking solutions, also in the case of tear down, the behavior is modified. If the B-V lightpath is torn down, the network controller has to account for the presence of the considered A-W connection. Thus, all the B-V spectrum resources will not be completely released. In particular, a slice of $12.5 \mathrm{GHz}$ contiguous to the $\mathrm{A}-\mathrm{W}$ resources will be maintained and reserved along E-P.

The effectiveness of the proposed superfilter technique in terms of overall network-spectrum savings has been assessed through networking simulations in [27], showing promising results particularly when the superfilter technique is combined with an additional technique called differentiated filter configuration [10].

\section{Filtering Cascade Effects}

To further detail the superfilter technique and the aforementioned reference scenarios, a possible transmission system is considered and a first analysis of filtering cascade effects is performed through simulations.

The considered optical signal consists of a polarization multiplexed QPSK (PM-QPSK) signal transmitted at a baud rate of $30 \mathrm{GS} / \mathrm{s}$, i.e., corresponding to a gross bit rate of $120 \mathrm{~Gb} / \mathrm{s}$. A TFP transmission technique with lowdensity parity-check (LDPC) codes and coherent detection is adopted here, as in [10].

A code rate of 9/10 is applied, thus obtaining an information rate after decoding [post forward error correction (FEC)] of $b_{r}=108 \mathrm{~Gb} / \mathrm{s}$. Furthermore, a concatenated outer code with $4 \%$ overhead is taken into account, to eliminate the error floor of LDPC codes. The net bit rate is then $103 \mathrm{~Gb} / \mathrm{s}$. The considered signal is particularly suitable for ITU-T flexigrid optical networks since it can be narrowfiltered at the transmitter and propagated, occupying only two frequency slices $\left(S_{m}=2\right.$, i.e., $\left.25 \mathrm{GHz}\right)$, achieving error-free operations for OSNR as low as $12 \mathrm{~dB}$. However, 

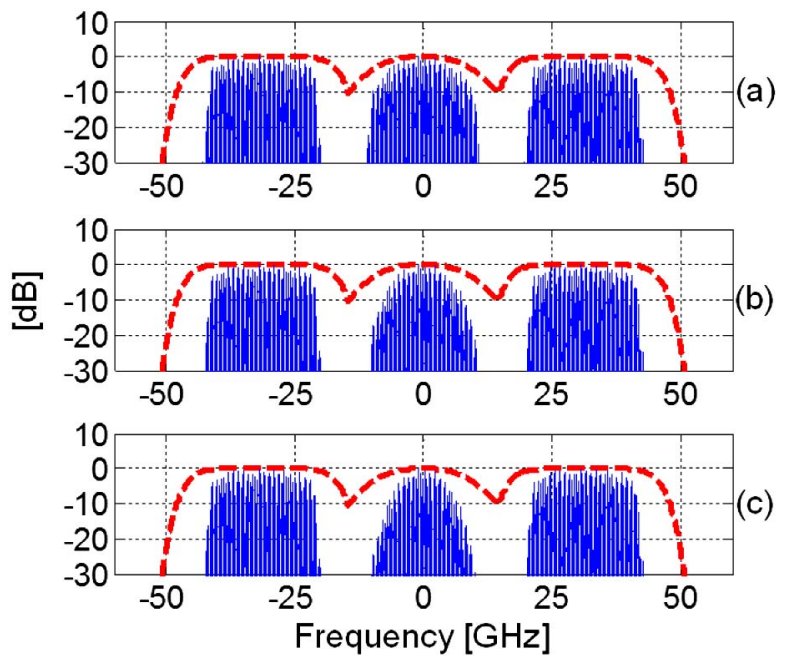

Fig. 2. Filter configuration (dashed line) and evolution of optical spectrum (a) after two, (b) four, and (c) six nodes in the first scenario. The central channel, traversing $25-\mathrm{GHz}$ filters, presents unacceptable QoT.

the propagation through a cascade of intermediate nodes including filters with $S=2$, given their nonideal filter shape, strongly affects the overall transmission (one filter per BV-OXC is assumed to be traversed).

Figures 2-4, show three optical carriers traversing BV-OXC filters and having different shapes. The shapes (dashed line) are obtained from measurements on a commercially available LCoS-based BV-OXC.

All three scenarios described in Section III are investigated.

In the first scenario, described in Fig. 1(b), a configuration of two spectrum slices of $12.5 \mathrm{GHz}$ is applied to the central lightpath A-W, corresponding to a spectrum allocation of $25 \mathrm{GHz}$, while three slices $(S=3,37.5 \mathrm{GHz})$
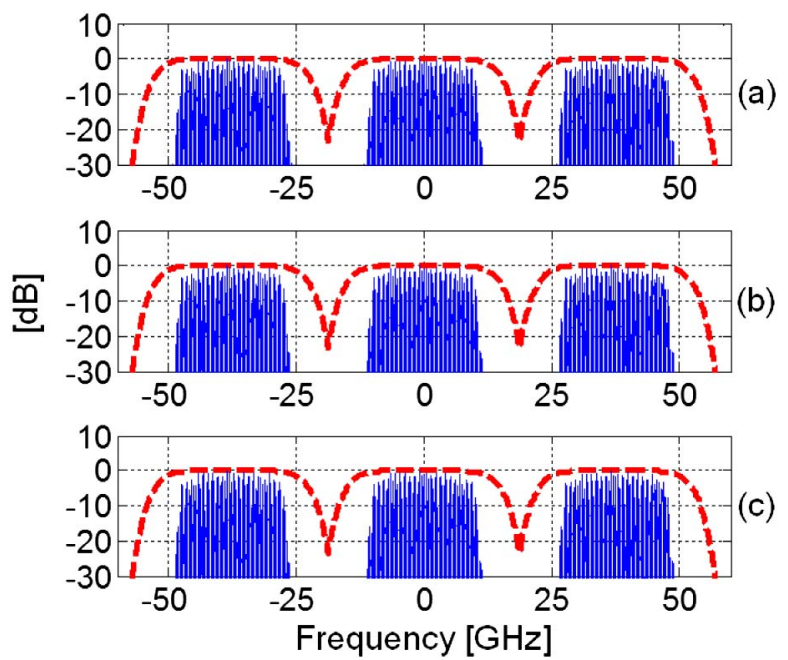

Fig. 3. Filter configuration (dashed line) and evolution of optical spectrum after (a) two, (b) four, and (c) six nodes in the second scenario. The central channel, traversing $37.5 \mathrm{GHz}$ filters, experiences adequate QoT.
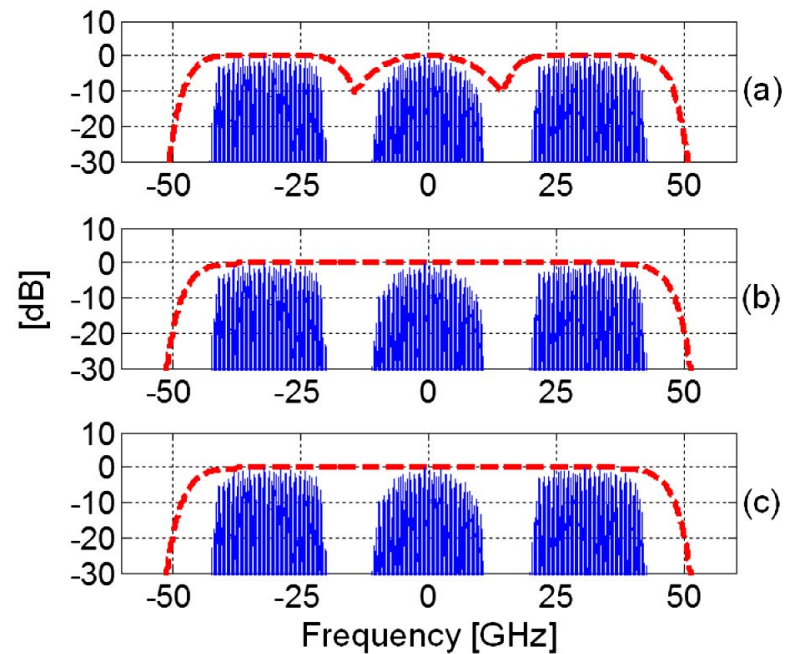

Fig. 4. Filter configuration (dashed line) and evolution of optical spectrum after (a) two, (b) four, and (c) six nodes in the third scenario, i.e., with superfilters. After the second node, a unique $100-\mathrm{GHz}$ filter is configured. The central channel experiences adequate QoT even if it reserves only $25 \mathrm{GHz}$.

are configured for both lightpaths B-V and C-Z (left and right channel). Figure 2 shows the optical spectrum evolution of the transmitted signal after (a) two nodes, (b) four nodes, and (c) six nodes. It is evident that, in all three cases, the transition bands are not negligible and the filter presents nonideal rectangular behavior. This is particularly evident in the case of the central filter with spectrum reservation $S=2(25 \mathrm{GHz})$, where the flat region is actually not present. The detrimental effects of this filtering cascade are also clear by observing the reduced optical signal spectrum after six traversed nodes [Fig. 2(c)].

The impact of the cascade of intermediate nodes has been measured in term of OSNR penalty at BER $=1 \mathrm{E}-5$. For this first scenario, the introduced penalty is shown in Fig. 5 (circles). Results show that around $1.5 \mathrm{~dB}$ of OSNR penalty per node is experienced for the first three traversed nodes with $S=2(25 \mathrm{GHz})$, while penalty further increases for each subsequent traversed node. A maximum number $N_{f}$ equal to 6 (with OSNR penalty as high as $12 \mathrm{~dB}$ ) of intermediate filters with $S=2$ is here admitted. Indeed,

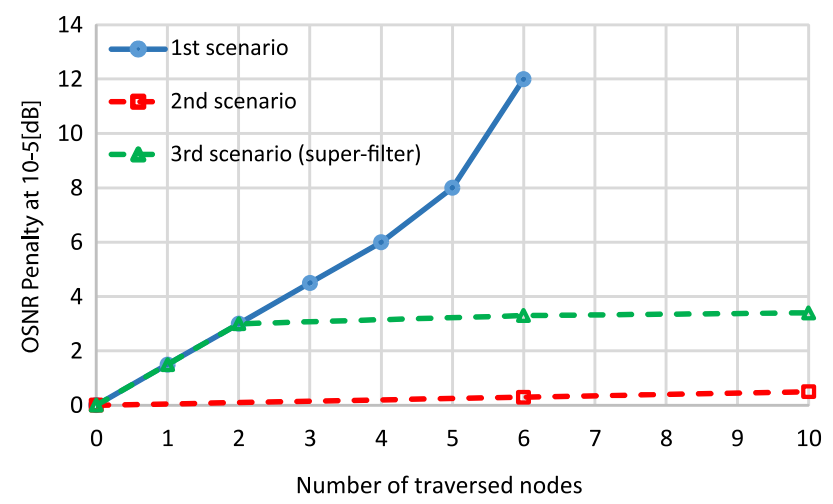

Fig. 5 . OSNR penalty at $\mathrm{BER}=1 \mathrm{E}-5$ due to filter cascade for the three considered scenarios. 
larger values of $N$ induce excessive distortions even with acceptable OSNR. To reduce these detrimental penalties and to enable the pass-through within a larger number of intermediate nodes, the second scenario described in Fig. 1(c) is then considered in Fig. 3.

In this second scenario, additional spectrum resources are reserved in all traversed nodes, and three spectrum slices of $12.5 \mathrm{GHz}$ are also reserved to lightpath $\mathrm{A}-\mathrm{W}$ [see (Fig. 3)]. Signal degradation along the filter cascade is reduced with respect to the previous scenario, and simulations show that only a $0.5-\mathrm{dB}$ penalty is experienced because of the pass-through of $N=6 \mathrm{BV}$-OXC with $S=3$ (37.5 GHz) as shown in Fig. 5 (squares). However, as shown in Fig. 3, this corresponds to a $50 \%$ increment of the spectrum reserved for lightpath $\mathrm{A}-\mathrm{W}$.

In the third scenario, described in Fig. 1(d), the proposed superfilter technique is applied. A unique filter configuration of value $S=8(100 \mathrm{GHz})$ among all signals is configured at node E [see Fig. 1(d)]. That is, a common filter is computed and applied to all three signals in transit D-G nodes, as shown in Fig. 4. Only $S=2$ is reserved for the central signal. After the detrimental effects introduced by the first two narrow filters, the subsequent cascade of filters is traversed in their flat region, without experiencing significant transmission degradation [(Fig. 5), triangles]. In this case, transmission always remains within the considered $N_{f}$ threshold.

\section{SDN IMPLEMENTATION OF SuPERFILTER}

A flexigrid network testbed including both control plane and data plane is utilized to validate the proposed superfilter technique. The testbed, derived from [10], reproduces the network shown in Fig. 1. It includes a specifically extended OF controller and OF-enabled node controllers operating on LCoS-based BV-OXCs.

\section{A. Control-Plane Implementation}

In this section, we focus on the control-plane implementation. Lightpaths B-V and C-Z are assumed to be already established. A request from source A to destination W arrives. The node controller at $\mathrm{A}$ sends a modified version of a PACKET_IN message suitably designed for optical connections (now called LIGHTPATH_IN) to the OF-controller to request both path computation and lightpath setup. The message is extended for flexigrid optical networks $[\underline{10}, \underline{23}, \underline{25}]$. Upon message reception, the OF-controller computes the path A-W. The OF-controller exploits a physicalparameter database (PPD) to gain knowledge of the expected QoT experienced by the lightpath along the network for the considered modulation format (PM-QPSK in this paper). The stateful OF-controller also exploits a LSPDB storing the attributes of existing active lightpaths, including their routes and related BV-OXC filtering configurations. This way, the OF-controller is able to account for filtering cascade effects as well as QoT parameters (e.g., OSNR) during path computation. When superfilters are not considered, the OF-controller relies only on available network resources (e.g., $25 \mathrm{GHz}$ along D-Q). Thus, the A-W request cannot be computed along $D-Q$ due to unacceptable QoT. On the other hand, by accounting for superfilters, the OF-controller successfully computes the path through those spectrum resources along D-Q. In particular, the OF-controller accounts for the presence of contiguous spectrum resources reserved along the path and it computes their expansions to incorporate the new request. The OF-controller then communicates to the node controllers at $\mathrm{A}, \mathrm{W}$, and to all the intermediate nodes along D-Q to perform the lightpath setup. In particular, the OF-controller sends FLOW_MOD messages extended for flexigrid networks to configure the optical switches, the transmitter, and the receiver. The FLOW_MOD messages directed to the transit nodes change the filter configuration identified by the nominal central frequency (n) and the channel width in terms of frequency slices (m). In this study, the nominal central frequency does not mean the actual central frequency of a specific signal, but means the central value of the frequency filter to be enforced at the BV-OXCequipped nodes. As shown in the Wireshark captures of Figs. 6 and 7, a spectrum reservation of $25 \mathrm{GHz}$ is sent by the OF-controller (IP: 10.0.0.49) to nodes A and W, as well as to nodes $\mathrm{D}$ and $\mathrm{Q}$. Then, nodes from $\mathrm{E}$ to $\mathrm{P}$ are configured with $S=8$, i.e., $100 \mathrm{GHz}$. In the latter case shown in Fig. 7, the novel flag SUPERFILTER is enclosed within the FLOW_MOD to bypass local filter contention and admission control and allow the filter overlap on the existing filters of B-V and C-Z lightpaths. This way, the path is successfully activated. Note that, as shown in the next section, no detrimental effects are induced on the adjacent B-V and C-Z lightpaths during filter adaptations since the LCoS configuration is not modified at those frequencies. If the $\mathrm{B}-\mathrm{V}$ lightpath is torn down, the OF-controller will not completely release the $\mathrm{B}-\mathrm{V}$ resources. In particular, a slice of $12.5 \mathrm{GHz}$ contiguous to the $\mathrm{A}-\mathrm{W}$ resources will be maintained along E-P. To this extent, the FLOW_MOD of type "delete" will include the SUPERFILTER flag and will

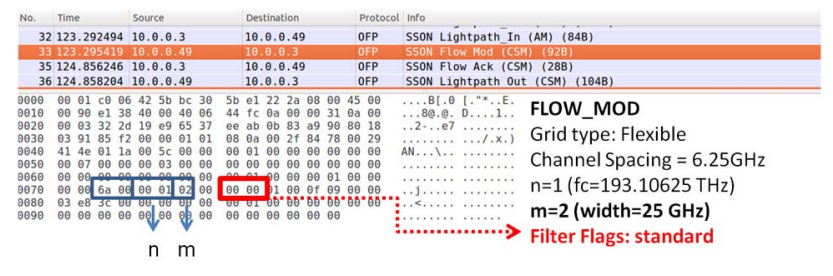

Fig. 6. Message capture at the node controller of ingress node A (IP: 10.0.0.3).

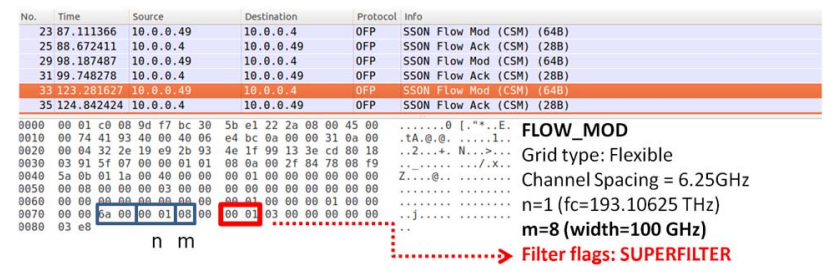

Fig. 7. Message capture at the node controller of the intermediate node E applying superfilters (IP: 10.0.0.4). 


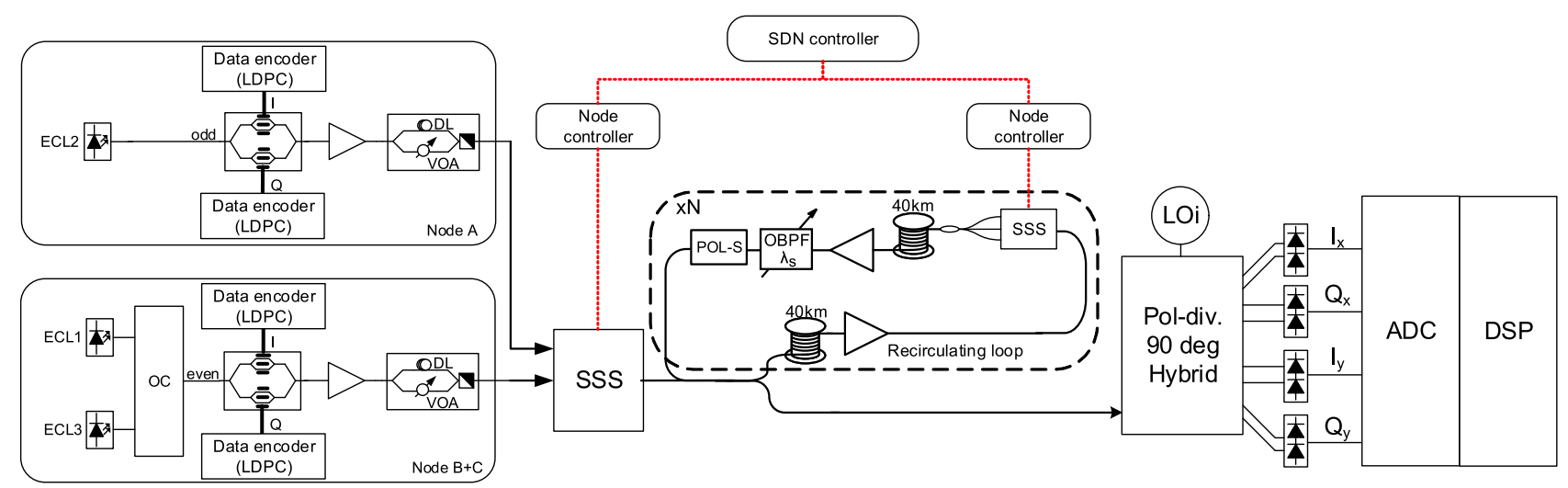

Fig. 8. BER measurements.

enclose the (n) and (m) values (different from those installed within the flow entry) corresponding to the portion of the filter to be released.

\section{B. Data-Plane Implementation}

In this section we focus on the data plane implementation. The experimental setup is shown in Fig. 8.

A TFP technique employing LDPC coding and off-line coherent detection is adopted as in [10]. Three carriers are generated by means of a $100-\mathrm{kHz}$ linewidth tunable laser source (TLS). In particular, the central carrier [the one generated at node $\mathrm{A}$ in (Fig. 1)] and the other two side carriers [generated at nodes $\mathrm{B}$ and $\mathrm{C}$ in (Fig. 1)] are separately modulated by using two integrated double-nested MachZehnder modulators (IQ-MZM). LDPC code is inserted by the encoder module shown in Fig. 8. Additionally, $30-\mathrm{Gb} / \mathrm{s}$ coded electrical data are applied to the in-phase (I) and the quadrature $(\mathrm{Q})$ ports of the modulators. This way, QPSK channels with a gross bit rate of $60 \mathrm{~Gb} / \mathrm{s}$ are obtained. The bit rate is then further doubled up to $120 \mathrm{~Gb} / \mathrm{s}$ per channel by emulating polarization multiplexing through a 50/50 beam splitter, an optical delay, and a polarization beam combiner (PBC). The rate of the used LDPC code is equal to $9 / 10$. A further $4 \%$ of redundancy is considered in order to allow error-free operation (after decoding, since the LDPC code has an intrinsic floor), thus providing a net bit rate of $103.8 \mathrm{~Gb} / \mathrm{s}$. A programmable BV-OXC is used to separately filter and multiplex the

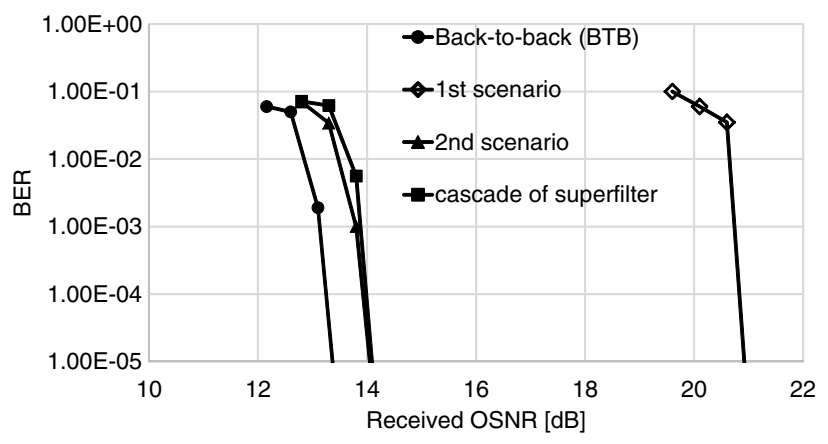

Fig. 9. Experimental testbed. channels following the scenario described in Fig. 1. A recirculating loop is used to reproduce the transmission of the channels through a cascade of nodes. The recirculating loop is comprised of two spans of 40-km-long standard singlemode fiber spools followed by an erbium-doped fiber amplifier (EDFA). A second programmable BV-OXC is used in the loop in order to specifically reproduce the cascading of several filters. A polarization scrambler (POL-S) in the loop emulates random polarization variation. All channels enter into the common port of the BV-OXC, which is programmed considering the three different scenarios described in Fig. 1.

The channels are then filtered and separated and then coupled again together at the BV-OXC output. BER for the back-to-back configuration (BTB) and the scenarios described in Fig. 1 are reported in Fig. 9. The reported BER measures are performed by averaging over 10 blocks of samples, each containing eight randomly selected code words per quadrature per polarization (with code rate equal to 9/10). BER values below $10^{-6}$ could not be reliably measured, such that the LDPC error floor (expected below $10^{-6}$ ) was not observed in the experimental setup. The system performance is also evaluated for all the scenarios, measuring the required OSNR to obtain error-free operation after a configured number of rounds into the fiber loop (i.e., number of traversed nodes).

The OSNR penalty with respect to the signal at the transmitter is depicted in Fig. 10. Considering the first scenario described in Fig. 1(b), an increasing penalty is

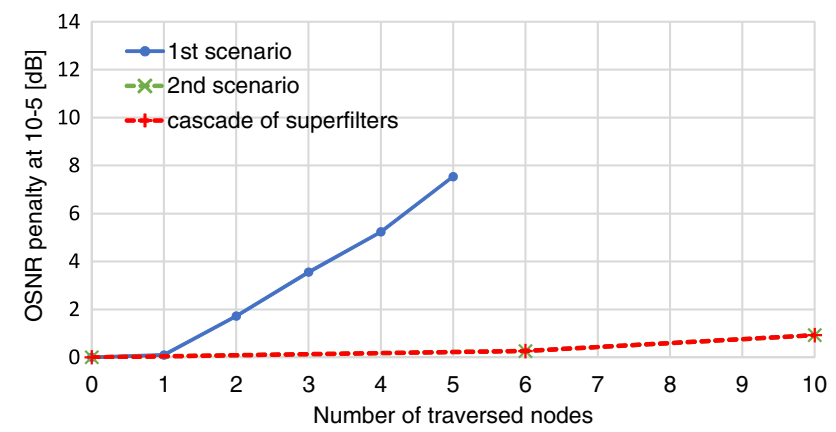

Fig. 10. OSNR penalty for the three scenarios in Figs. 1(b)-1(d) (as cascade of superfilters). 


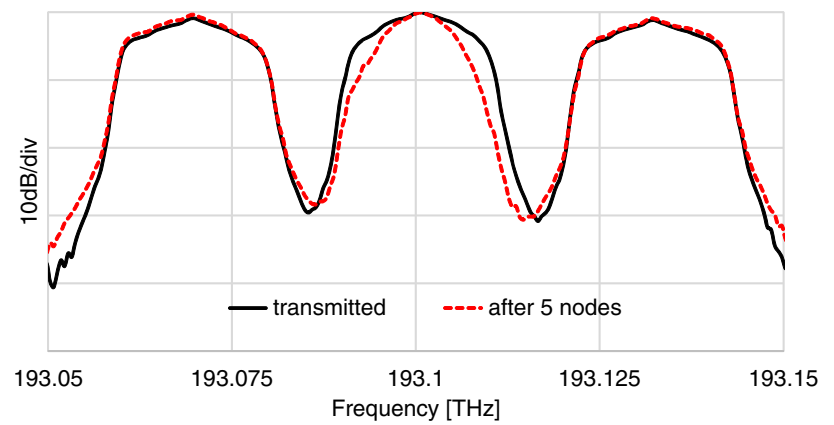

Fig. 11. Optical spectrum of the channels at the transmitter and after five loop rounds (i.e., five traversed nodes) for the scenario of Fig. 1(b).

measured when the number of traversed nodes increases. Error-free operation cannot be achieved for more than five traversed nodes, and a penalty of $7.5 \mathrm{~dB}$ is measured after five nodes (in agreement with the simulative results reporting a penalty of $8 \mathrm{~dB}$ after five nodes). The optical spectrum of the channels at the transmitter and after five nodes (i.e., five fiber loop rounds) is reported in Fig. 11, showing a clear filtering effect for the central channel.

In Fig. 10, the measurements for the second scenarios of Fig. 1(c) are also reported. Also in this case, simulative results are confirmed, showing minor penalty only after 10 nodes.

Due to hardware constraints (only two BV-OXCs are employed in the testbed), the case of Fig. 1(d) is reported, focusing only on the cascade of superfilters. In particular, only one filter is traversed before the signal enters the recirculating loop. Results show a penalty of about $1 \mathrm{~dB}$ after 10 traversed nodes, thus confirming the successful behavior of the proposed superfilter technique.

\section{CONCLUSIONS}

In this study, the novel superfilter technique for flexigrid EONs was introduced to improve spectral efficiency and avoid over-reservation of spectrum resources during path computation. In particular, the technique enables different lightpaths, also having different source-destination pairs, to be corouted within the same flat region of a single filter. This way, detrimental filtering cascade effects are reduced and, as a consequence, more efficient spectrum utilization is achieved.

Simulative transmission results showed that one spectrum slice out of three, dedicated to a single lightpath, can be saved when the superfilter technique is applied. The technique has been successfully implemented on a SDN architecture, which showed to be extremely suitable for supporting differentiated filter configurations decoupled from head-end lightpath configurations. Moreover, OF extensions for superfilters were proposed and successfully demonstrated. The SDN architecture was successfully applied to a flexigrid network testbed including a recirculating loop to accurately reproduce filtering cascade effects.
The proposed superfilter technique opens the way for a new family of efficient impairment-aware routing and spectrum-assignment solutions and reoptimization procedures, where lightpaths are routed trying to maximize the spectrum contiguity along common network segments.

\section{ACKNOWLEDGMENT}

This work was partially supported by the EU-FP7 IDEALIST project under grant agreement number 317999. This paper is an extended version of the work presented in [1].

\section{REFERENCES}

[1] F. Paolucci, F. Fresi, A. Castro, L. Velasco, F. Cugini, N. Sambo, A. Giorgetti, L. Potì, and P. Castoldi, "Filter optimization in SDN-based flexgrid networks," in Optical Fiber Communication Conf. (OFC), 2014.

[2] M. Jinno, B. Kozicki, H. Takara, A. Watanabe, Y. Sone, T. Tanaka, and A. Hirano, "Distance-adaptive spectrum resource allocation in spectrum-sliced elastic optical path network," IEEE Commun. Mag., vol. 48, no. 8, pp. 138-145, Aug. 2010.

[3] O. Gerstel, M. Jinno, A. Lord, and S. Yoo, "Elastic optical networking: A new dawn for the optical layer?" IEEE Commun. Mag., vol. 50, no. 2, pp. 12-20, Feb. 2012.

[4] "Spectral grids for WDM applications: DWDM frequency grid," ITU-T Recommendation G.694.1, 2012.

[5] O. Gonzales de Dios, R. Casellas, F. Zhang, and X. Zi, "Framework and requirements for GMPLS based control of flexi-grid DWDM networks," IETF draft-ietf-ccamp-flexigrid-fwk-00, Oct. 2013.

[6] E. Ip and J. Kahn, "Fiber impairment compensation using coherent detection and digital signal processing," J. Lightwave Technol., vol. 28, no. 4, pp. 502-519, 2010.

[7] A. Morea, J. Renaudier, A. Ghazisaeidi, O. Bertran-Pardo, and $\mathrm{T}$. Zami, "Impact of reducing channel spacing from $50 \mathrm{GHz}$ to $37.5 \mathrm{GHz}$ in fully transparent meshed networks," in Optical Fiber Communication Conf. (OFC), Mar. 2014.

[8] M. Filer and S. Tibuleac, "N-degree ROADM architecture comparison: Broadcast-and-select versus route-and-select in $120 \mathrm{~Gb} / \mathrm{s}$ DP-QPSK transmission systems," in Optical Fiber Communication Conf. (OFC), Mar. 2014.

[9] S. Poole, S. Frisken, M. A. Roelens, and C. Cameron, "Bandwidth-flexible ROADMs as network elements," in Optical Fiber Communication Conf. (OFC), 2011.

[10] N. Sambo, G. Meloni, F. Paolucci, F. Cugini, M. Secondini, F. Fresi, L. Potì, and P. Castoldi, "Programmable transponder, code and differentiated filter configuration in elastic optical networks," J. Lightwave Technol. vol. 32, pp. 2079-2086, 2014.

[11] Open Networking Foundation, 2013. [Online]. Available: https://www.opennetworking.org/working-groups/opticaltransport.

[12] C. Saradhi and S. Subramaniam, "Physical layer impairment aware routing (PLIAR) in WDM optical networks: Issues and challenges," IEEE Commun. Surv. Tutorials, vol. 11, no. 4, pp. 109-130, 2009.

[13] S. Azodolmolky, M. Klinkowski, E. Marin, D. Careglio, J. Pareta, and I. Tomkos, "A survey on physical layer impairments aware routing and wavelength assignment algorithms 
in optical networks," Comput. Netw., vol. 53, no. 7, pp. 926-944, 2009.

[14] N. Sambo, M. Secondini, F. Cugini, G. Bottari, P. Iovanna, F. Cavaliere, and P. Castoldi, "Modeling and distributed provisioning in $10-40-100-\mathrm{Gb} / \mathrm{s}$ multirate wavelength switched optical networks," J. Lightwave Technol. vol. 29, no. 9, pp. 1248-1257, May 2011.

[15] A. Nag, M. Tornatore, M. Liu, and B. Mukherjee, "Routing and wavelength assignment in WDM networks with mixed line rates," in Cross-Layer Design in Optical Networks. Springer, 2013, pp. 53-77.

[16] S. Peng, R. Nejabati, and D. Simeonidou, "Impairment-aware optical network virtualization in single-line-rate and mixedline-rate WDM networks," J. Opt. Commun. Netw., vol. 5, no. 4, pp. 283-293, 2013.

[17] L. Velasco, A. Jirattigalachote, M. Ruiz, P. Monti, L. Wosinska, and G. Junyent, "Statistical approach for fast impairment-aware provisioning in dynamic all-optical networks," J. Opt. Commun. Netw., vol. 4, no. 2, pp. 130-141, 2012.

[18] A. G. Rahbar, "Review of dynamic impairment-aware routing and wavelength assignment techniques in all-optical wavelength-routed networks," IEEE Commun. Surv. Tutorials, vol. 14, no. 4, pp. 1065-1089, 2012.

[19] F. Paolucci, F. Cugini, A. Giorgetti, N. Sambo, and P. Castoldi, "A survey on the path computation element (PCE) architecture," IEEE Commun. Surv. Tutorials, vol. 15, no. 4, pp. 1819-1841, 2013.

[20] S. Tibuleac and M. Filer, "Transmission impairments in DWDM networks with reconfigurable optical add-drop multiplexers," J. Lightwave Technol., vol. 28, no. 4, pp. 557-598, 2010.
[21] B. Kozicki, H. Takara, Y. Tsukishima, T. Yoshimatsu, K. Yonenaga, and M. Jinno, "Experimental demonstration of spectrum-sliced elastic optical path network (SLICE)," Opt. Express, vol. 18, no. 21, pp. 22105-22118, 2010.

[22] F. Paolucci, F. Cugini, N. Hussain, F. Fresi, and L. Poti, "Openflow-based flexible optical networks with enhanced monitoring functionalities," in European Conf. and Exhibition on Optical Communication (ECOC), 2012.

[23] J. Zhang, J. Zhang, Y. Zhao, H. Yang, X. Yu, L. Wang, and X. $\mathrm{Fu}$, "Experimental demonstration of openflow-based control plane for elastic lightpath provisioning in flexigrid optical networks," Opt. Express, vol. 21, no. 2, pp. 1364-1373, 2013.

[24] N. Sambo, F. Paolucci, F. Cugini, M. Secondini, L. Poti, G. Berrettini, G. Meloni, F. Fresi, G. Bottari, and P. Castoldi, "Software defined code-rate-adaptive terabit/s based on time-frequency packing," in Optical Fiber Communication Conf. (OFC), 2013.

[25] L. Liu, W.-R. Peng, R. Casellas, T. Tsuritani, I. Morita, R. Martínez, R. Muñoz, and S. Yoo, "Design and performance evaluation of an openflow-based control plane for softwaredefined elastic optical networks with direct-detection optical OFDM (DDO-OFDM) transmission," Opt. Express, vol. 22, no. 1, pp. 30-40, 2014.

[26] G. Colavolpe and T. Foggi, "Time-frequency packing for highcapacity coherent optical links," IEEE Trans. Commun., vol. 62, no. 8, pp. 2986-2995, Aug. 2014.

[27] A. Castro, F. Cugini, L. Velasco, and P. Castoldi, "On the benefits of differentiating the filter configurations in flexigrid optical networks," in European Conf. and Exhibition on Optical Communication (ECOC), 2014. 